\title{
ORIENTACIONES PARA RESOLVER CASOS DE TEORÍA DEL DELITO
}

\author{
Guidelines for resolving cases of crime theory
}

Manuel VIDAURRI ARÉCHIGA

\section{Sumario:}

I. Comentarios introductorios. II. Importancia de la legislación, doctrina y jurisprudencia. III. Consejos y sugerencias a modo de principios. IV. Orientaciones para resolver casos penales. V. Fuentes.

Resumen: El autor hace sugerencias a los estudiantes de Derecho penal para solucionar casos de teoría del delito. Se trata de orientaciones básicas que complementan las explicaciones de los profesores.

Palabras clave: Derecho penal, teoría del delito.

Abstract: The author makes suggestions to students of criminal law theory to solve crime cases. These are basic guidelines that complement the explanations of teachers.

Keywords: Criminal law. Theory of crime.

\section{COMENTARIOS INTRODUCTORIOS}

Las presentes orientaciones tienen una pretensión muy específica: auxiliar al estudiante de teoría del delito a resolver casos penales, ${ }^{2}$ proponiéndole una metodología que le guíe en ese propósito. En cualquier actividad humana, si se quieren los mejores resultados, siempre es mejor seguir una metodología que prescindir de la misma. Son más los inconvenientes de proceder sin método que las ventajas que se desprenden de seguir una serie de pasos dirigi-

\footnotetext{
1 Doctor en Derecho por la Universidad de Sevilla. Profesor Investigador del Departamento de Derecho de la División de Derecho, Política y Gobierno de la Universidad de Guanajuato. Pertenece al Sistema Nacional de Investigadores (Nivel II) y cuenta con perfil PROMEP. Miembro del Cuerpo Académico: Ciencia Penal, Estado de Derecho y Derechos Humanos.

Una versión de este texto formará parte del libro que sobre la teoría general del delito viene preparando el autor.

${ }^{2}$ Como sugerencias bibliográficas sobre resolución de casos penales se encuentran las siguientes: AGUDELO BETANCUR, Nódier, Casos de Derecho penal, Editorial Temis, Bogotá, 1990; ESER, Albin y BJÖRN Burkhardt, Derecho penal. Cuestiones fundamentales de la teoría del delito sobre la base de casos de sentencias, Editorial Colex, Madrid, 1995; FELLINI, Zullita, Resolución de casos de Derecho penal, Ediciones Depalma, Buenos Aires, 2004; KERN, Eduard, Guía para el tratamiento de casos de Derecho penal, Ediciones Depalma, Buenos Aires, 1968; MAQUEDA ABREU, María Luisa y Patricia Laurenzo Copello, El Derecho penal en casos, tercera edición, Editorial Tirant lo Blanch, Valencia, 201; ROMERO, Gladys, Casos de Derecho penal. Manual práctico; Ediciones Depalma, Buenos Aires, 1992.
} 
dos al logro del objetivo determinado. Otra cosa es que, luego de probarla, la metodología no resulte adecuada, lo cual tiene solución: adoptamos otra.

Aprender teoría del delito es una tarea que exige dedicación y esfuerzo. La utilización de casos penales, generalmente hipotéticos, es una técnica frecuente en nuestras Facultades y Escuelas de Derecho ya que permite al estudiante poner en práctica los aspectos teóricos de la asignatura. Consecuentemente, en este capítulo ponemos a disposición del estudiante una serie de consejos y sugerencias metodológicas dirigidas a facilitar el análisis y resolución de los casos sugeridos en clase.

Esta guía no sustituye, en modo alguno, al profesor. Indiscutiblemente, su apoyo y orientación será fundamental por lo que la asistencia a las sesiones en el aula resultan insustituibles. La investigación y el estudio individual así como el trabajo de análisis en grupo, son otras actividades que no deben descuidarse.

Por otra parte, a lo largo de su proceso formativo el estudiante de teoría del delito deberá completar las siguientes tres tareas:

- Conocimiento del código penal que se aplicará al caso concreto.

- Familiarizarse con una serie de conceptos y teorías que la doctrina jurídico-penal ha venido construyendo a lo largo del tiempo.

- Desarrollar un método para analizar (y resolver) los casos de teoría del delito que nos presenten.

\section{I.1. CONOCIMIENTO DEL CÓDIGO PENAL QUE SE APLICARÁ AL CASO CONCRETO}

Conocer un código es igual a decir que lo hemos estudiado, lo hemos comentado, lo hemos discutido. Ahora que, como es bien sabido, coexisten en nuestro país tantos códigos penales como entidades federativas lo componen, además de uno de alcance federal y uno más para el Distrito Federal, por lo que resulta aconsejable conocer lo mejor posible el del propio Estado y, en lo posible, el Código Penal Federal, por aquello de las figuras típicas que solo son de competencia federal. Desde luego, realizar análisis comparativos entre los códigos puede ser altamente ilustrativo. Un consejo más: procurar conseguir siempre el código penal vigente, siendo conveniente que el mismo sea obtenido directamente en la instancia correspondiente del poder legislativo. ${ }^{3}$ Muy ventajosos es el hecho de que ahora todo el orden jurídico nacional pueda ser consultados en línea. ${ }^{4}$

\section{I.2. FAMILIARIDAD CON LOS CONCEPTOS Y TEORÍAS JURÍDICO-PENALES}

Generalmente, los programas de estudio de la carrera de Derecho consideran una serie de cursos de Derecho penal a través de los cuales se estudia la teoría de la norma, la teoría del delito y, finalmente, la teoría de la pena y las medidas de seguridad. En cada uno de estos momentos se van mostrando al estudiante los conceptos y teorías fundamentales e imprescindibles para el conocimiento y manejo de la disciplina. Aprender las principales teorías así

\footnotetext{
3 Se recomienda obtener siempre la última versión oficial misma que, generalmente, estará a disposición del público en los portales o páginas web de los congresos locales y federal.

4 www.ordenjuridico.gob.mx; también: www.scjn.gob.mx, 10 de diciembre de 2013. 
como identificar y aplicar las diferentes categorías conceptuales propias del derecho penal servirán enormemente, tanto en la etapa de formación, como en la praxis profesional.

Por lo demás, dado que el Derecho positivo y la doctrina sufren transformaciones con cierta frecuencia, resulta aconsejable procurar mantenerse al día, bien acudiendo con frecuencia a seminarios, talleres y conferencias sobre la materia, así como por la adquisición de material bibliográfico básico y especializado. La consulta de revistas electrónica, la consulta de acervos científicos especializados o jurisprudenciales deberá convertirse en una constante, tanto en la vida estudiantil como en la profesional.

I.3. Desarrollar un método para analizar (Y Resolver) los Casos de teoría del DELITO QUE NOS PRESENTEN

Cualquier actividad humana, incluyendo la intelectual, requiere de un método para desarrollarlas con provecho y eficacia. Sobre este particular, no debemos olvidar que los penalistas contamos con un método propio: la dogmática penal. Ya en otro lugar hemos señalado que la dogmática penal es el método utilizado por los penalistas para imponerse del contenido del Derecho penal positivo5. Sin embargo, aquí nos referimos a algo relativamente diferente; pensamos en una metodología para resolver casos penales, esto es una serie de pasos a seguir para encontrar una solución, la cual estará sustentada en la dogmática penal, entendida ahora como el compendio de teorías jurídico- penales elaboradas con fines esclarecedores del problema penal considerado globalmente.

\section{IMPORTANCIA DE LA LEGISLACIÓN, DOCTRINA Y JURISPRUDENCIA}

Conforme vaya avanzando en los estudios profesionales, el estudiante se irá dando cuenta de que el aprendizaje del Derecho se logra con tenacidad, constancia y concentración. También notará que en ese trabajo cotidiano juegan un papel importantísimo la legislación, la doctrina y la jurisprudencia. ${ }^{6}$

- Conocer la ley equivale a verificar el qué y el cómo se regula un determinado aspecto de la vida en sociedad; la ley dice que camino debe seguirse y hasta donde llegan las posibilidades del individuo con respecto al ejercicio de su libertad individual respecto de las de otras personas.

- Imponerse de las aportaciones científicas de los investigadores y tratadistas de la ciencia jurídica, esto es, conocer la doctrina sobre cualquier materia, permitirá identificar los problemas concretos pero también las diferentes propuestas de solución.

- Enterarse de los contenidos propios de la jurisprudencia nos acercará a la praxis jurídica, lo que no es poca cosa; el derecho penal es saber practico y su concreción se logra al momento en que los jueces aplican lo dispuesto por la ley.

5 VIDAURRI ARÉCHIGA, Manuel, Introducción al Derecho penal, Editorial Oxford University Press, MéxiCo, 2012, p. 30.

${ }^{6}$ FELLINI, Zulita, Resolución de casos de Derecho penal, Op. Cit., p. 10, menciona al respecto: "Antes de aplicar un texto legal es necesario hacer un exhaustivo estudio del mismo a través de las fuentes de conocimiento: doctrina y jurisprudencia en sus acepciones amplias". 


\section{CONSEJOS Y SUGERENCIAS A MODO DE PRINCIPIOS}

Reconocer que nunca un caso es igual a otro

Parece una simpleza decir lo anterior, sin embargo es necesario repetirlo. Cada caso tiene sus propias características; serán distintos los sujetos que cometen el delito y los que lo padecen; será siempre distinto el lugar, tiempo, modo y ocasión. Y también es posible que la ley aplicable sea distinta, bien porque se haya modificado en el lapso transcurrido entre un caso y otro, bien porque se trata del ámbito local o federal, o bien porque se refiera a un código penal de otra entidad federativa.

\section{Antes de emitir alguna opinión debe estudiarse concienzudamente el caso}

Los penalistas deben ser, en un primer momento, mesurados en sus opiniones, las que deben basarse en la ley, orientarse por la doctrina más actual y consistente, y someterse a la jurisprudencia aplicable al caso en cuestión. Visto así, deslizar una opinión a la ligera puede traer consecuencias indeseables. Luego, se aconseja leer el caso más de una vez, sin dejar de analizar cualquier detalle por menor que parezca y nunca agregar elementos inexistentes. Todos los hechos que se dan en el caso se los supone absolutamente probados, con lo cual es innecesario detenerse a examinar la certeza de ellos. ${ }^{7}$

\section{Prohibido imaginar que ocurrió algo que el caso no dice}

Si no se observa este principio, es posible que se encuentre una solución, pero a un caso distinto y no al que nos han presentado.

\section{Aceptar que no hay soluciones únicas}

Ya se verá a lo largo de la actividad profesional, que un caso puede tener más de una respuesta, dependiendo de quien haga el estudio y del grado de profundidad con el que el caso mismo sea analizado. Como bien indica Gladys Romero, la solución a la que se llegue no es tan importante como la fundamentación que de ella se haga. ${ }^{8}$ Más aún, la solución del caso dependerá igualmente de la teoría del delito utilizada, de modo que al decir que no hay una solución única debemos agregar que tampoco hay una sola teoría del delito.

\section{Preparar esquemas de solución provisionales}

A cada paso que se vaya dando en el análisis del caso es pertinente construir un esquema de solución. Cada problema particular del caso en cuestión deberá ser resuelto, y es preferible detenerse en cada aspecto, aunque esto represente mayor esfuerzo. La suma de esquemas y sus respectivas soluciones abonará a la conclusión final.

\footnotetext{
7 ROMERO, Gladys, Casos de Derecho penal. Manual práctico, Op. Cit., p. 3; también así, FELLINI, Zulita, Resolución de casos penales, Op. Cit., p. 10.

8 Ibidem., p. 9. 
Seguir un orden en el análisis del caso

Naturalmente de esto hablamos cuando nos referimos a contar con una metodología. En este momento, pensamos en seguir un orden analítico al modo siguiente: primero, hacer la confrontación de los hechos con lo establecido en la ley, luego realizar una revisión de las opiniones teóricas que sobre el problema jurídico se hayan formulado y, por último, verificar la opinión jurisprudencial que pudiera haber al respecto. Siguiendo estos pasos se alcanzará una visión integral del problema.

\section{Procurar construirse una opinión propia en torno al caso}

Las posturas doctrinales o las tesis jurisprudenciales pueden no ser las más razonables o las más lógicas. Puede incluso tratarse de opiniones ya trascendidas por virtud del signo de los tiempos o por aportaciones teóricas más acabadas. Por eso mismo, el analista deberá buscar construir y formular su opinión misma que, finalmente, será la que defienda frente a sus contrapartes.

Considerar en todo momento la vigencia y observancia de los principios estructurales del Derecho penal

El Derecho penal de corte democrático se construye sobre la base de una serie de principios limitadores del ius puniendi, todos los cuales deben ser observados sin excepción por el analista del caso penal. Si bien es cierto, uno de estos principios (el de legalidad) es básico, también lo son el de presunción de inocencia, culpabilidad, exclusiva protección de benes jurídicos, materialidad, por mencionar expresamente algunos.

\section{ORIENTACIONES PARA RESOLVER CASOS PENALES}

Según un reconocido autor:

La solución de un caso penal consiste en trasladar a un suceso concreto la decisión general contenida en el texto legal. Para ello es necesario valerse del sistema de análisis de la teoría del delito. Se llega a la solución mediante un proceso mental que comienza con una intuición global de las soluciones posibles. Tales intuiciones son depuradas a través del análisis de los elementos del hecho que permiten la aplicación de los conceptos de la dogmática. La intuición inicial de la solución, suele estar fuertemente influida por las representaciones del que juzga, respecto de los fines de la pena. ${ }^{9}$

Según pensamos, la teoría del delito es un instrumento conceptual que permite la aplicación racional de la ley penal a los casos concretos. En cierto modo, la teoría del delito induce a la realización de tres juicios sucesivos: uno sobre la conducta del autor, otro sobre su contrariedad con la norma y un tercero referido a la posibilidad de responsabilizar personalmente al autor por el hecho antijurídico realizado; poniendo atención, nos damos cuenta que estos juicios se corresponden con las categorías básicas que componen la teoría del delito:

\footnotetext{
9 BACIGAlUPO, Enrique, Técnica de solución de casos penales, Editorial Hammurabi, Buenos Aires, 1998, p.
} 82, citado por FELLINI, Zulita, Resolución de casos penales, Op. Cit., p. 10. 
tipicidad, antijuridicidad y culpabilidad; ${ }^{10}$ por supuesto, también deberá considerarse la punibilidad, como consecuencia jurídica del delito y la cual posee reglas de aplicación que igualmente deben ser tomadas en cuenta.

Hasta aquí hemos delineado algunos elementos a seguir; primeramente con unas consideraciones generales sobre los presupuestos propios del estudiante, seguidos de una serie de principios básicos y luego con algunas reflexiones sucintas en torno a la ley, la doctrina y la jurisprudencia. A continuación, nos ocuparemos de los pasos que deben darse para la resolución de un caso penal, siguiendo en lo general los esquemas que, por su lado, han propuesto Fellini Zulita, Maqueda Abreu y Laurenzo Copello. ${ }^{11}$

Obviamente, al estudiar un caso penal debe tenerse a la mano el código penal con el que piensa resolverse el mismo. De la misma manera, será útil contar con las notas de clase y el libro de estudio.

\section{IV.1. ASPECTOS GENERALES}

- ¿Qué hizo el sujeto activo del delito? ¿mató, robo, violó? al responder esta interrogante se estará identificando la conducta penalmente relevante.

- Determinar si se trata de una conducta perpetrada por acción u omisión del sujeto activo. En este punto, deberá descartarse alguna causa de exclusión, como pueden ser la fuerza física irresistible, actos reflejos, estados de inconsciencia absoluta, etc.

- Especificar los tipos penales que puedan resultar aplicables. El objetivo es verificar que los hechos encuadren en algún tipo penal; es lo que se conoce como subsunción. En este punto, no se prejuzga la responsabilidad personal del autor.

- Precisar si se trata de un delito de lesión o de peligro, común o especial; es decir, que tipo de delito es el cometido.

- Identificar si el tipo penal se ha cometido dolosa o culposamente.

\section{IV.2. ACCIÓN DOLOSA}

- Si se trata de una acción dolosa, analizar estos aspectos:

TIPICIDAD:

- Elementos objetivos: acción, sujetos, objeto, resultado, relación de causalidad.

- Elementos subjetivos: Dolo directo o eventual; la presencia de algún tipo de error.

\footnotetext{
${ }^{10}$ En este sentido MAQUEDA ABREU, María Luisa y Patricia Laurenzo Copello, El Derecho penal en casos, Op. Cit., p. 255.

${ }^{11}$ FELLINI, Zulita, Resolución de casos penales, Op. Cit., p. 13 y siguientes; así como las aportaciones de Maqueda Abreu, María Luisa y Patricia Laurenzo Copello, El Derecho penal en casos, Op. Cit., p. 255 y siguientes. 


\section{ANTIJURIDICIDAD:}

Verificar la presencia de alguna causa de justificación o de error:

- Legítima defensa.

- Estado de necesidad.

- Ejercicio de un Derecho.

- Cumplimiento de un deber.

- e) Error.

\section{CULPABILIDAD:}

Poniendo atención en sus presupuestos:

- Comprobar la imputabilidad del autor.

- Conocimiento de la antijuridicidad.

- Capacidad de culpabilidad.

- Exigibilidad de otra conducta.

- Error de prohibición.

\section{PUNIBILIDAD:}

En este aspecto habrá de verificarse si el tipo penal establece algún requisito para la aplicación de la pena que sea diverso al injusto o a la culpabilidad. Si se prevé una excusa absolutoria o alguna condición objetiva de punibilidad.

TENTATIVA:

Que puede ser idónea o inidónea, acabada o inacabada. Igualmente si hubo desistimiento por parte del sujeto activo.

AUTORÍA Y PARTICIPACIÓN:

- Autoría: directa, mediata y coautoría.

- Participación: inducción o complicidad.

CONCURSO:

De leyes o concurso de delitos -real o ideal-. 


\section{IV.3. ACCIÓN CULPOSA O IMPRUDENTE}

Si se trata de una acción culposa, analizar estos aspectos:

TIPICIDAD:

- Asegurarse que el tipo penal admite esta forma de realización.

- Analizar el disvalor de la acción y el disvalor del resultado.

- Precisar la infracción al deber de cuidado.

- Revisar lo concerniente a la relación de causalidad.

ANTIJURIDICIDAD:

Analizar lo relativo a las causas de justificación.

CULPABILIDAD:

- Capacidad de culpabilidad.

- Exigibilidad de otra conducta.

- Conocimiento del contenido de la norma de cuidado.

- Error de prohibición.

PUNIBILIDAD:

La que se determine para el tipo penal cometido.

AUTORÍA Y PARTICIPACIÓN:

Determinación del autor; las formas de participación estará en función de la del autor.

CONCURSO:

De leyes y de delitos.

IV.4. OMISIÓN DOLOSA

- Tratándose de delitos de omisión dolosa, revisar estos puntos:

TIPICIDAD

Elementos objetivos: 
1. DELITOS PROPIOS DE OMISIÓN:

- Situación generadora del deber.

- Acreditar la omisión.

- Verificar la capacidad de actuación del sujeto activo.

2. DELITOS IMPROPIOS DE OMISIÓN:

- Comprobar la producción del resultado de un delito de comisión.

- Verificar que ha intentado impedir la producción del resultado.

- Establecer que la realización de la acción omitida hubiera evitado el resultado.

- Acreditar la capacidad para actuar.

- Determinar la posición de garante (sus fuentes).

ELEMENTOS SUBJETIVOS:

- El dolo.

- Conocimiento de la situación generadora del deber de actuar.

- Error.

Por lo que hace a la antijuridicidad, culpabilidad, punibilidad, tentativa, autoría y participación, la punibilidad y el concurso, aplica lo señalado en el apartado II.

IV.5. OMISIÓN CULPOSA O IMPRUDENTE

Si el delito se cometió por omisión imprudente o culposa, la única diferencia con el de comisión consiste en que el autor no infringió el cuidado debido con un acto positivo sino omitiendo. Los demás elementos son coincidentes.

\section{FUENTES}

AGUDELO BETANCUR, Nódier, Casos de Derecho penal, Editorial Temis, Bogotá, 1990.

BACIGALUPO, Enrique, Técnica de solución de casos penales, Editorial Hammurabi, Buenos Aires, 1998.

ESER, Albin y BJÖRN Burkhardt, Derecho penal. Cuestiones fundamentales de la teoría del delito sobre la base de casos de sentencias, Editorial Colex, Madrid, 1995.

FELLINI, Zullita, Resolución de casos de Derecho penal, Ediciones Depalma, Buenos Aires, 2004; 
KERN, Eduard, Guía para el tratamiento de casos de Derecho penal, Ediciones Depalma, Buenos Aires, 1968.

MAQUEDA Abreu, María Luisa y Patricia Laurenzo Copello, El Derecho penal en casos, tercera edición, Editorial Tirant lo Blanch, Valencia, 2011.

ROMERO, Gladys, Casos de Derecho penal. Manual práctico; Ediciones Depalma, Buenos Aires, 1992.

VIDAURRI ARÉCHIGA, Manuel, Introducción al Derecho penal, Editorial Oxford University Press, México, 2012. 